\title{
Interventions aimed at addressing unplanned hospital readmissions in the U.S.: A systematic review
}

\author{
Alva O. Ferdinand ${ }^{1}$, Ohbet Cheon*2, Abdulaziz T. Bako ${ }^{3}$, Bita A. Kash ${ }^{1,2}$ \\ ${ }^{1}$ Texas A\&M University School of Public Health, United States \\ ${ }^{2}$ Houston Methodist Research Institute, United States \\ ${ }^{3}$ Richard M. Fairbanks School of Public Health, Indiana University-Purdue University Indianapolis, United States
}

Received: October 31, 2018

DOI: $10.5430 /$ jha.v8n1p16

\author{
Accepted: December 2, $2018 \quad$ Online Published: December 14, 2018 \\ URL: https://doi.org/10.5430/jha.v8n1p16
}

\begin{abstract}
One of the policy mechanisms aimed at improving population health through health care delivery is the Hospital Readmissions Reduction Program (HRRP) as outlined in the Affordable Care Act. Although numerous procedural and behavioral interventions have been implemented, the empirical evidence of the efficacy of these interventions is mixed and specific to certain patient segments. This review aimed to systematically assess studies of hospital interventions to reduce 30-day readmissions for specific diseases and populations. Following the PRISMA review checklist, searches were conducted from January 2000 to August 2018 in the MEDLINE and EMBASE databases using terms such as "patient readmission", "readmit" and "re-hospitalization" in conjunction with disease terms such as "asthma", "chronic obstructive pulmonary disease (COPD)" and "pneumonia". Of 3,806 articles identified, 45 were included after a 3-step inclusion process. The age group most frequently considered among the studies was the 65 age cohort. Multidisciplinary collaborative interventions were most frequently effective for the subset of elderly, female, Caucasian, and heart failure patients. Interventions involving patient or family education delivered before and after care were most effective for racial minority, elderly, COPD, and heart failure patients. Telephone follow-up, tele-homecare, and medication reconciliation were largely found to be successful in reducing readmissions. Major gaps exist in identifying successful interventions for reducing 30-day readmissions among patients who sought treatment for sepsis, stroke, and replacement of the hip or knee. Our findings indicate an opportunity for researchers to further study, and for healthcare organizations to implement, more well-informed interventional strategies to reduce readmissions.
\end{abstract}

Key Words: Hospital readmission, Interventions, Population, Systematic review

\section{INTRODUCTION}

Unplanned hospital readmissions render implications for population health, as they are generally thought of as indicators of substandard health care delivery quality, ${ }^{[1]}$ and are often associated with adverse patient outcomes. ${ }^{[2]}$ Moreover, unplanned readmissions occur at a great cost to private and public insurers in the U.S., with estimations in 2011 that Medicaid, private insurance, and Medicare paid 18\%, 20\%, and 58\% of all readmission-associated costs in the U.S. respectively. ${ }^{[3]}$ To address patient safety and quality concerns, and to empower more informed health care decision-making on the part of patients, the Centers for Medicare and Medicaid Services (CMS) began publicly reporting 30-day readmission rates for heart failure, myocardial infarction, and pneumonia in $2010 .{ }^{[4]}$ In response to the economic burden of hospital readmissions, policymakers incorporated the es-

*Correspondence: Ohbet Cheon; Email: ocheon@ houstonmethodist.org; Address: Houston Methodist Research Institute, 6560 Fannin St. 1150, Houston TX 77030, United States. 
tablishment of the CMS Hospital Readmissions Reduction Program (HRRP) into the Affordable Care Act as a means of incentivizing hospitals to engage in efforts that would reduce preventable readmissions. ${ }^{[5-8]}$ Starting in October 2012, CMS was empowered to impose financial penalties on hospitals with higher-than-expected readmissions for the same conditions that the agency began publicly reporting on in 2010. ${ }^{[8]}$ Since then, CMS has expanded the applicable conditions to include chronic obstructive pulmonary disease (COPD), total hip arthroplasty (THA), total knee arthroplasty (TKA), and coronary artery bypass graft (CABG).

Numerous procedural and behavioral interventions have been conceptualized, implemented, and examined for effectiveness vis-à-vis hospital readmission reductions. These interventions include, but are not limited to, improved oral and written communication between providers and family members,,${ }^{[9-11]}$ post-discharge support teams, ${ }^{[10]}$ multidisciplinary provider teams, ${ }^{[12]}$ coordinated care ${ }^{[13-15]}$ homebased care, ${ }^{[16]}$ emphasis on medication reconciliation and adherence, ${ }^{[17,18]}$ patient hotlines, follow-up appointments patient education and engagement, ${ }^{[19,20]}$ tele-monitoring, ${ }^{[21]}$ and discharge planning, ${ }^{[12,22,23]}$ among others. The evidence centered on the efficacy of these interventions is quite mixed. For example, some researchers have generally found that discharge planning interventions are effective in reducing hospital readmissions, ${ }^{[23,24]}$ while others have found inconsistent or moderate effects of similar interventions on readmission rates. $^{[12,22]}$ Findings from research on the effectiveness of transition of care interventions (TOCs) are also mixed. Generally, TOCs are sets of actions taken by clinical staff to ensure patient coordination and continuity of care as patients are shifted between various hospital units (e.g. an emergency department to an intensive care unit) and/or different locations (e.g. hospital to home). ${ }^{[15]}$ While some researchers have found that TOCs have been associated with significant decreases in hospital readmission rates, ${ }^{[13,17]}$ others have not found such an association. ${ }^{[25]}$ Moreover, studies that have examined the efficacy of medication reconciliation interventions have also provided conflicting findings. ${ }^{[12,26,27]}$

To further add complexity, many of the study samples in the literature examining interventions targeted toward reducing hospital readmissions have been specific to certain sub-populations. For example, several studies have tested interventions on the elderly population only ${ }^{[28-31]}$ while others have exclusively focused on joint replacement, ${ }^{[10]}$ heart failure, ${ }^{[13,19-21,32]}$ stroke, ${ }^{[25]}$ and Medicare ${ }^{[33]}$ patients. Thus, the breadth and strength of the evidence-base on interventions to address hospital readmission is difficult to ascertain because of heterogeneity in the patient populations, interventions, clinical settings, and implementation strategies that

Published by Sciedu Press have been examined and adopted. This heterogeneity also makes efforts to keep abreast of current findings on the part of hospital managers and clinicians especially arduous.

Therefore, the purpose of this study was to conduct a systematic review that will outline the state of the literature across interventions, populations, and various diagnoses. We were interested in any articles that examined the relationship between a targeted intervention and hospital readmissions on the following commonly occurring diagnoses: heart failure, acute myocardial infarction (AMI), COPD, bronchitis and asthma, pneumonia and other respiratory tract infections, sepsis, stroke, and TKA or THA. Our work supplements previous reviews of the readmission intervention literature that have narrowly focused on care delivered in patients' homes, ${ }^{[16]}$ nurse-assisted case management, ${ }^{[14]}$ medication reconciliation, ${ }^{[26,27,34]}$ self-management programs, ${ }^{[19]}$ and TOC interventions. ${ }^{[15,25,35]}$ In our study, we were broadly interested in determining what kinds of interventions are most promising for reducing hospital readmissions among patients with the aforementioned commonly occurring diagnoses. We were also interested in determining where major gaps exist in the literature. To identify these gaps, we set out to quantify the number of studies on specific interventions and their effectiveness on readmissions across various diagnoses. Overall, our study will be useful to hospital managers, clinicians, case managers, public health personnel, and researchers interested in reducing the occurrence of short-term hospital readmissions and improving care associated with index hospitalizations in the U.S.

\section{METHODS}

Following the PRISMA review checklist, ${ }^{[36]}$ we used a systematic review methodology to identify and extract information from articles that examined the efficacy of interventions aimed at reducing short-term hospital readmissions. We included articles published from January 2000 to August 2018. To be as comprehensive as possible, we enlisted the assistance of a professionally trained library science expert. We searched the MEDLINE and EMBASE databases for search terms such as "patient readmission", "readmit", "repeated hospitalization" and "re-hospitalization" to capture studies done on readmission. Moreover, we used terms such as "myocardial infarction", "heart failure", "asthma", "replacement", "chronic obstructive lung disease", "pneumonia", "stroke", "cerebrovascular disease", "cerebrovascular accident", "intracranial hemorrhage" and "respiratory tract infections" to capture articles that specifically examined readmissions among diagnoses commonly associated with shortterm readmissions for which hospitals are being scrutinized. We only considered English-language, U.S.-based empirical 
publications that appeared in peer-reviewed journals. Because we were exclusively focused on the evidence base for the impact of various interventions on readmissions, we ex- cluded policy briefs, letters to the editor, and governmental reports outlining the general problem.

\section{Step 1: Title Review}

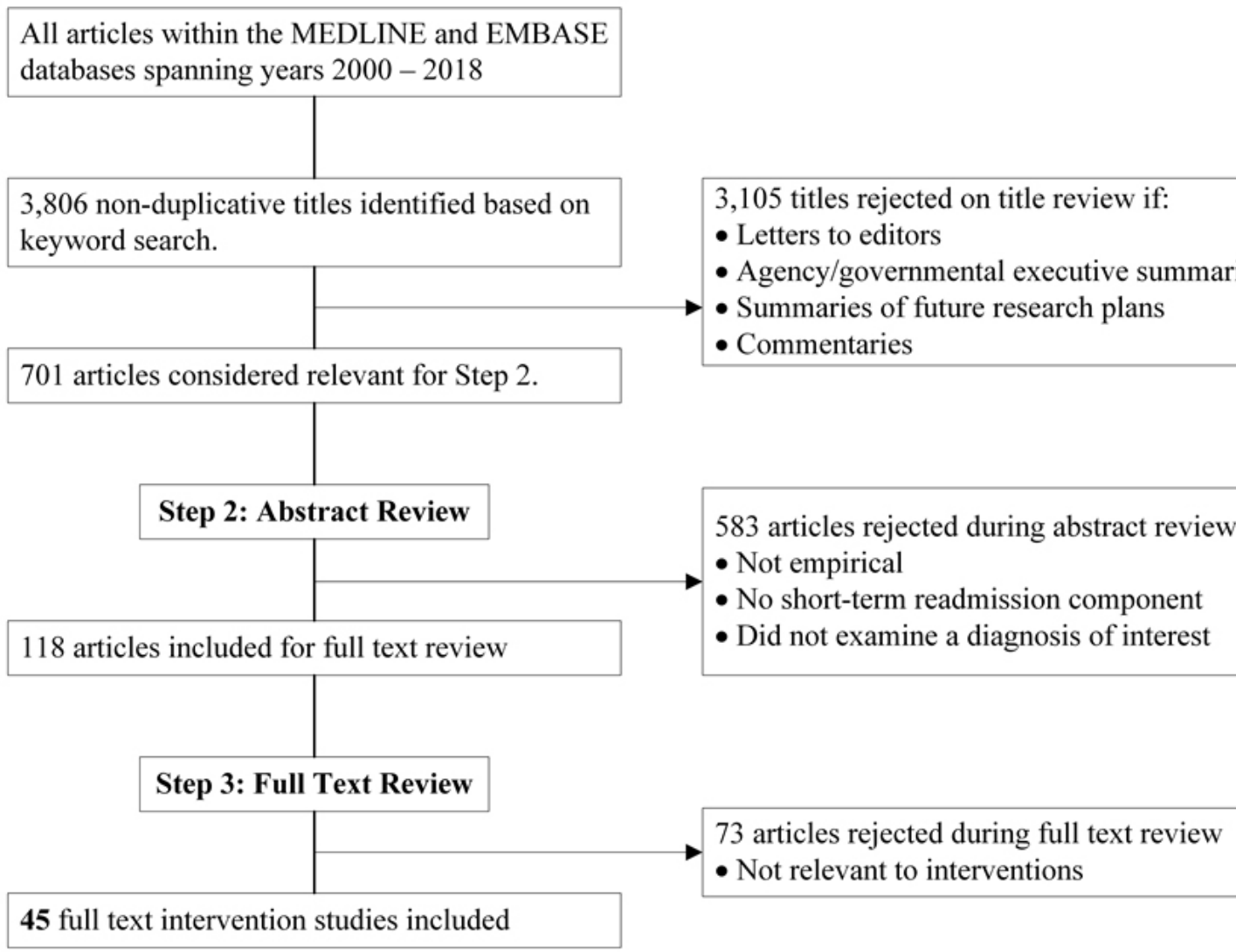

Figure 1. Identification of literature examining the effectiveness of interventions aimed at hospital readmission

Our keyword search identified 3,806 articles through our search of the aforementioned databases. We used a 3-step inclusion process outlined in Figure 1. In the first step, two independent reviewers (A.T.B. and A.O.F.) examined article titles and each flagged articles for elimination that did not indicate a focus on short-term readmission and one of the diagnoses of interest. The comprehensive nature of our search terms picked up articles that did not fit squarely in the scope of our study. For example, our initial search captured many articles that examined the association of specific operational or pharmaceutical interventions on short-term readmissions, ${ }^{[37-39]}$ and the use of various readmission metrics in predicting unplanned readmissions. ${ }^{[40-42]}$ We sought a high level of sensitivity in this first step in that we erred on the side of inclusion. Letters to editors, governmental summaries, and commentaries were excluded in this step. Consequently, if either reviewer chose to include an article on the basis of the title, it was selected for abstract review in step 2. We included a total of 701 titles $(18 \%)$ once this step was completed.

The second step involved screening the abstracts of the 701 articles identified in step 1. During this phase of our literature search, we focused on a high level of specificity by excluding articles that were not empirical studies and did not examine one of the diagnoses of interest. Disagreements or uncertainty about inclusion or exclusion was reconciled by group discussion among the authors. We included a total of 118 articles once this step was completed. Because of our interest in identifying procedural and behavioral interventions that are effective in reducing short-term readmissions, we conducted a third step in which we identified studies that explicitly examined readmission outcomes subsequent to the implementation of a non-clinical or medicinal intervention. 
We included a total of 45 articles in this systematic review. These 45 studies represented 66 individual analyses because several papers presented two or more separate analyses that fit our inclusion criteria.

Next, we systematically classified all included studies by using a standard coding sheet specifically developed for this study. For each intervention study, we collected information on traits of the sample, such as the sample size, and whether race, gender, and age were considered in the study. We additionally collected information on whether patients with specific types of insurance coverage were included in the study (e.g. Medicare or Medicaid), and whether a study considered patients' educational attainment in their examination of the effectiveness of an intervention. In examining interventions used, we also distinguished between studies that examined one intervention independently and those that examined bundled interventions (for example studies that examined an intervention that included telephone follow-up, discharge planning, and collaboration among providers). We differentiated interventions based on 12 categories on the basis of existing literature, ${ }^{[43,44]}$ and coded them with the intervention name, description, clinic setting, study design. We additionally gathered information on whether each study found a beneficial association between the intervention(s) under study and short-term readmission (i.e. whether there was a reduction in readmission), a non-significant association, or an association that yielded mixed results. Studies were rated using a modified scheme from the Oxford Centre for Evidence-based Medicine to rate evidence quality from 1 (high) to 5 (low) quality. ${ }^{[45]}$

\section{Results}

We included a total of 45 articles in this review. These 45 studies represented 66 individual interventions because several papers presented two or more separate interventions that fit inclusion criteria. The number of articles examining the association of interventions on short-term hospital readmissions increased over time with one article published before 2000 and 35 published between 2011 and 2016. Sample sizes varied considerably among the studies with one study utilizing a sample of less than 50 participants and 14 studies examining the effects of interventions with more than 1,000 sample participants. The age group most frequently considered among the studies was the 65 or older age cohort. Eleven studies focused on individuals aged 18 to 64 . Gender was also considered in most studies. 35 studies considered patients' gender in their models. Most studies, however, did not consider individual educational attainment or income levels of their samples in their analyses. Additionally, 28 of the 45 articles did not consider or provide information about individual insurance status in their studies (see Table 1).

Table 1. Descriptive findings of selected intervention studies ( $\mathrm{N}=45$ studies)

\begin{tabular}{|c|c|c|}
\hline Variables & Number of articles & $(\%)$ \\
\hline \multicolumn{3}{|l|}{ Year } \\
\hline - $<2000$ & 1 & $2 \%$ \\
\hline - 2000-2005 & 3 & $7 \%$ \\
\hline - 2006-2010 & 6 & $13 \%$ \\
\hline - 2011-2016 & 35 & $78 \%$ \\
\hline \multicolumn{3}{|l|}{ Sample Size } \\
\hline - $<50$ & 1 & $2 \%$ \\
\hline - $51-100$ & 3 & $7 \%$ \\
\hline - 101-500 & 19 & $42 \%$ \\
\hline - $501-1,000$ & 8 & $18 \%$ \\
\hline - $1,001-22,000$ & 14 & $31 \%$ \\
\hline \multicolumn{3}{|l|}{ Insurance Type } \\
\hline - Medicare & 16 & $36 \%$ \\
\hline - Medicaid & 7 & $16 \%$ \\
\hline - Private & 7 & $16 \%$ \\
\hline - Not Included & 28 & $62 \%$ \\
\hline \multicolumn{3}{|l|}{ Race } \\
\hline - Caucasian & 25 & $56 \%$ \\
\hline - Black & 24 & $53 \%$ \\
\hline - Hispanic & 9 & $20 \%$ \\
\hline - Other $^{*}$ & 20 & $44 \%$ \\
\hline - Not Included & 14 & $31 \%$ \\
\hline \multicolumn{3}{|l|}{ Age } \\
\hline - $<2$ & 2 & $4 \%$ \\
\hline - 2-18 years & 2 & $4 \%$ \\
\hline - 18-64 years & 11 & $24 \%$ \\
\hline - $\geq 65$ years & 27 & $60 \%$ \\
\hline - Not included & 3 & $7 \%$ \\
\hline \multicolumn{3}{|l|}{ Education } \\
\hline - Included (Less than college) & 3 & $7 \%$ \\
\hline - Not Included & 42 & $93 \%$ \\
\hline \multicolumn{3}{|l|}{ Income } \\
\hline - Included & 0 & $0 \%$ \\
\hline - Not Included & 45 & $100 \%$ \\
\hline \multicolumn{3}{|l|}{ Gender } \\
\hline - Included & 35 & $78 \%$ \\
\hline - Not Included & 10 & $22 \%$ \\
\hline \multicolumn{3}{|c|}{$\begin{array}{l}\text { Study reported a statistically beneficial relationship between the } \\
\text { intervention and short-term readmission }\end{array}$} \\
\hline - Yes & 20 & $44 \%$ \\
\hline - No & 23 & $51 \%$ \\
\hline - Partially $^{* *}$ & 2 & $4 \%$ \\
\hline
\end{tabular}

A list and description of the interventions aimed toward reducing short-term hospital readmissions can be found in Kash et al.'s study. ${ }^{[43,44]}$ A breakdown of the number of studies examining various strategies and the number finding beneficial, null, or mixed effects can be found in Table 2 . Of the 11 studies that examined educational strategies, in which patients and/or family members are educated on the diagnosis in question in an effort to enhance knowledge and 
involvement in care, 4 found beneficial results of such an intervention, while 6 studies did not find beneficial reductions in short-term readmissions following an education-based intervention. Of the 9 studies that examined strategies centered around collaboration (i.e. interventions that involved activities conducted by a multidisciplinary team), 3 found beneficial associations between the intervention and readmission, while 6 reported null findings. The three studies that found a beneficial relationship between the collaborative interventions and readmission reductions had samples that were primarily made up of elderly, female, and Cau- casian heart failure patients. These sample characteristics were quite different to the samples of the 6 studies reporting null findings. These studies' participants primarily consisted of children under 18 who had been diagnosed with asthma, COPD, bronchiolitis, or pneumonia. The findings indicate that the efficacy of interventions on short-term readmission reduction can be highly dependent on diagnosis and patient characteristics.

The detailed information for the studies finding beneficial outcomes in readmission reductions are listed in Table 3.

Table 2. Descriptive analysis of intervention studies ( $N=45$ articles, 66 interventions)

\begin{tabular}{|c|c|c|c|c|c|}
\hline Type of Intervention & Description of Intervention & Count & $\begin{array}{l}\text { Positive } \\
\text { Results (\%) }\end{array}$ & $\begin{array}{l}\text { Negative or } \\
\text { Not-significant } \\
\text { Results }(\%)\end{array}$ & $\begin{array}{l}\text { Mixed } \\
\text { Results }(\%)^{*}\end{array}$ \\
\hline 1 (Collaboration) & $\begin{array}{l}\text { A variety of activities of a multidisciplinary team } \\
\text { or group that includes diverse professions }\end{array}$ & $9(14 \%)$ & $3(33 \%)$ & $6(67 \%)$ & 0 \\
\hline 2 (Home visits) & $\begin{array}{l}\text { Physical visits by healthcare provider(s) to } \\
\text { patient's home; Regular home visits by } \\
\text { profession(s) }\end{array}$ & $1(2 \%)$ & $1(100 \%)$ & 0 & 0 \\
\hline 3 (Telephone follow-up) & $\begin{array}{l}\text { Use of telephone or videophone for } \\
\text { provided-initiated communication initiated after } \\
\text { discharge }\end{array}$ & $5(8 \%)$ & $3(60 \%)$ & $2(40 \%)$ & 0 \\
\hline 4 (Education) & $\begin{array}{l}\text { Patient-directed education or coaching to } \\
\text { diagnosis or treatment rationale in order to } \\
\text { increase patient/family's knowledge and } \\
\text { enhance their involvement in care }\end{array}$ & $11(17 \%)$ & $4(36 \%)$ & $6(55 \%)$ & $1(9 \%)$ \\
\hline $\begin{array}{l}5 \text { (Medication } \\
\text { reconciliation) }\end{array}$ & $\begin{array}{l}\text { Medication reconciliation or special education } \\
\text { conducted by pharmacist and/or nurse, which is } \\
\text { aimed at improving medication understanding or } \\
\text { adherence before discharge }\end{array}$ & $5(8 \%)$ & $3(60 \%)$ & $1(20 \%)$ & $1(20 \%)$ \\
\hline 6 (Discharge planning) & $\begin{array}{l}\text { Formalizing an approach to prepare for } \\
\text { discharge; Establishing a transitional care plan } \\
\text { before discharge }\end{array}$ & $5(8 \%)$ & $2(40 \%)$ & $3(60 \%)$ & 0 \\
\hline $\begin{array}{l}7 \text { (Follow-up } \\
\text { appointment) }\end{array}$ & $\begin{array}{l}\text { Scheduling a follow-up visit with patients during } \\
\text { their hospital stay }\end{array}$ & $1(2 \%)$ & $1(100 \%)$ & 0 & 0 \\
\hline 8 (Tele-monitoring) & $\begin{array}{l}\text { Use of remote technology designed for patients } \\
\text { to transmit objective measures of health status } \\
\text { with or without connected subjective assessment }\end{array}$ & $5(8 \%)$ & $3(60 \%)$ & $2(40 \%)$ & 0 \\
\hline $\begin{array}{l}9 \text { (Guideline } \\
\text { implementation) }\end{array}$ & $\begin{array}{l}\text { Adoption of and adherence to guidelines } \\
\text { including rules or regulations about clinical } \\
\text { treatment; Compliance with guidelines regarding } \\
\text { staffing like work-hour restrictions }\end{array}$ & $8(12 \%)$ & $3(38 \%)$ & $4(50 \%)$ & $1(13 \%)$ \\
\hline 10 (Rehabilitation) & $\begin{array}{l}\text { Patient-directed rehabilitation efforts that are not } \\
\text { entirely diagnosis specific but aimed at } \\
\text { improving functional status }\end{array}$ & $2(3 \%)$ & $1(50 \%)$ & $1(50 \%)$ & 0 \\
\hline $\begin{array}{l}11 \text { (Clinical, medical } \\
\text { device) }\end{array}$ & $\begin{array}{l}\text { Implementation of certain drug or surgery } \\
\text { treatment; Use of an innovative medical device } \\
\text { in a surgery or medical care to improve clinical } \\
\text { outcomes }\end{array}$ & $7(11 \%)$ & $3(43 \%)$ & $4(57 \%)$ & 0 \\
\hline $\begin{array}{l}12 \text { (In-hospital } \\
\text { management) }\end{array}$ & $\begin{array}{l}\text { Hospital wards, staffed by doctors, nurses and } \\
\text { other health professionals for diagnostic } \\
\text { assessment, therapy, rehabilitation, and } \\
\text { placement of patients to intensify post discharge } \\
\text { care, identify effective community services and } \\
\text { enhance primary care access }\end{array}$ & $7(11 \%)$ & $2(29 \%)$ & $5(71 \%)$ & 0 \\
\hline
\end{tabular}

Note. Mixed results signify studies that rendered different results, for example by hospital sites or by diagnoses. Description of intervention reprinted with permission from Kash et al. ${ }^{[44]}$ 
Table 3. Characteristics of studies finding beneficial associations between interventions and short-term readmissions

\begin{tabular}{|c|c|c|c|c|c|c|c|}
\hline Authors & Type & $\begin{array}{l}\text { Intervention } \\
\text { Description }\end{array}$ & Diagnosis & Outcome & Significance & Takeaway points & $\begin{array}{l}\text { Quality of } \\
\text { Evidence }\end{array}$ \\
\hline $\begin{array}{l}\text { Akosah et al., } \\
2002\end{array}$ & 1,12 & $\begin{array}{l}\text { Short-term, } \\
\text { aggressive-intervention } \\
\text { heart failure clinic } \\
\text { (HFC) }\end{array}$ & Heart failure & $\begin{array}{l}\text { Readmission, } \\
\text { mortality rate }\end{array}$ & $\begin{array}{l}\text { Compared to patients followed by their } \\
\text { primary care physicians, there was a } 77 \% \\
\text { relative risk reduction for } 30 \text {-day hospital } \\
\text { readmission, and a lower rate of } \\
\text { readmissions at } 90 \text { days and } 1 \text { year in the } \\
\text { intervention group. Additionally, } \\
\text { combined hospital readmission and } \\
\text { mortality rate at } 90 \text { days ( } 10 \% \text { vs. } 30 \%, p \\
<.018 \text { ) and } 1 \text { year ( } 21 \% \text { vs. } 43 \%, p<.02) \\
\text { was lower in patients referred to the HFC } \\
\text { (intervention group). }\end{array}$ & $\begin{array}{l}\text { HFC patients experienced fewer } \\
\text { re-hospitalizations and improved } \\
\text { event-free survival compared to } \\
\text { patients followed up by their } \\
\text { primary care physicians. }\end{array}$ & 3 \\
\hline $\begin{array}{l}\text { Amarasingham } \\
\text { et al., } 2013\end{array}$ & 8 & $\begin{array}{l}\text { Intensive set of } \\
\text { evidence-based } \\
\text { interventions, using } \\
\text { electronic medical } \\
\text { record (EMR) }\end{array}$ & Heart failure & $\begin{array}{l}\text { Readmission for } \\
\text { any cause and to } \\
\text { any hospital } \\
\text { within } 30 \text { days of } \\
\text { discharge }\end{array}$ & $\begin{array}{l}\text { The unadjusted readmission rate declined } \\
\text { from } 26.2 \% \text { in the pre-intervention period } \\
\text { to } 21.2 \% \text { in the post-intervention period ( } p \\
=.01 \text { ), a decline that persisted in adjusted } \\
\text { analyses (adjusted OR [AOR] }=0.73 \text {; } \\
95 \% \text { CI } 0.58 \text { to } 0.93, p=.01 \text { ). }\end{array}$ & $\begin{array}{l}\text { An EMR-enabled strategy that } \\
\text { targeted scarce care transition } \\
\text { resources to high-risk HF patients } \\
\text { significantly reduced the } \\
\text { risk-adjusted odds of readmission. }\end{array}$ & 2 \\
\hline $\begin{array}{l}\text { Baker et al., } \\
2015\end{array}$ & 1 & $\begin{array}{l}\text { Inter-hospital } \\
\text { collaborative approach }\end{array}$ & Heart failure & $\begin{array}{l}\text { 7-day } \\
\text { post-discharge } \\
\text { follow-up rates } \\
\text { and 30-day } \\
\text { readmission }\end{array}$ & $\begin{array}{l}\text { During the intervention period, } \\
\text { unadjusted readmissions decreased } \\
\text { significantly ( } 29.0 \% \text { to } 27.3 \% ; p<.001) \text {. }\end{array}$ & $\begin{array}{l}\text { See You in } 7 \text { Collaborative } \\
\text { participation was associated with } \\
\text { significantly lower } 30 \text {-day } \\
\text { readmissions }\end{array}$ & 2 \\
\hline $\begin{array}{l}\text { Benzo et al., } \\
2016\end{array}$ & 4,5 & $\begin{array}{l}\text { Health coaching and } \\
\text { action plan for the use } \\
\text { of antibiotics and oral } \\
\text { steroids }\end{array}$ & COPD & $\begin{array}{l}\text { 30-day and 90-day } \\
\text { readmissions }\end{array}$ & $\begin{array}{l}\text { The absolute risk reductions of } \\
\text { COPD-related rehospitalization in the } \\
\text { intervention arm compared with the } \\
\text { control arm were } 7.5 \%(p=.01) \text { at } 30 \text {-day } \\
\text { postdischarge, and } 11.0(p=.02) \text { at } \\
\text { 90-day postdischarge. }\end{array}$ & $\begin{array}{l}\text { Health coaching can be an } \\
\text { effective intervention designed to } \\
\text { reduce COPD readmissions }\end{array}$ & 1 \\
\hline $\begin{array}{l}\text { Dean et al., } \\
2006\end{array}$ & 9 & $\begin{array}{l}\text { Pneumonia guideline } \\
\text { implementation }\end{array}$ & $\begin{array}{l}\text { Respiratory } \\
\text { failure and } \\
\text { sepsis from } \\
\text { pneumonia }\end{array}$ & $\begin{array}{l}\text { 30-day all-cause } \\
\text { mortality, length } \\
\text { of hospital stay, } \\
\text { and readmission } \\
\text { rate }\end{array}$ & $\begin{array}{l}\text { A 1-SD increase }(10 \%) \text { in guideline } \\
\text { compliance was associated with a lower } \\
\text { readmission rate after guideline } \\
\text { implementation [odds ration }(95 \% \mathrm{CI})= \\
0.86(0.78 \text { to } 0.96), p=.006 \text { ]. }\end{array}$ & $\begin{array}{l}\text { Improved clinical outcomes were } \\
\text { associated with pneumonia } \\
\text { guideline utilization. }\end{array}$ & 4 \\
\hline $\begin{array}{l}\text { Jones et al., } \\
2011\end{array}$ & 10 & $\begin{array}{l}\text { A pilot program for } \\
\text { provision of Post Acute } \\
\text { Care (PAC) }\end{array}$ & $\begin{array}{l}\text { Neurological } \\
\text { diagnosis } \\
\text { (commonly } \\
\text { stroke) }\end{array}$ & $\begin{array}{l}\text { Post-discharge } \\
\text { readmission and } \\
\text { emergency } \\
\text { department visits }\end{array}$ & $\begin{array}{l}\text { The } 30 \text {-day readmission rate for adult day } \\
\text { program participants was significantly } \\
\text { lower than that for non-participants; } 6.1 \% \\
\text { vs. } 22.2 \%(p=.05) \text {. }\end{array}$ & $\begin{array}{l}\text { An expanded adult day program } \\
\text { may represent a viable } \\
\text { Transitional Care Model for } \\
\text { selected patients and a feasible } \\
\text { alternative to skilled nursing } \\
\text { facility and home health care for } \\
\text { PAC. }\end{array}$ & 4 \\
\hline $\begin{array}{l}\text { Lichtman } \\
\text { et al., } 2009\end{array}$ & 9 & $\begin{array}{l}\text { Joint Commission } \\
\text { Primary Stroke Center } \\
\text { certification program }\end{array}$ & Ischemic stroke & $\begin{array}{l}\text { Mortality, 30-day } \\
\text { mortality, and } \\
\text { 30-day } \\
\text { readmission }\end{array}$ & $\begin{array}{l}\text { In-hospital mortality (OR, } 0.93 ; 95 \% \mathrm{CI} \text {, } \\
0.90 \text { to } 0.96 \text { ), } 30 \text {-day mortality (OR, } 0.92 \text {; } \\
95 \% \mathrm{CI}, 0.87 \text { to } 0.96 \text { ), } 30 \text {-day readmission } \\
\text { (hazard ratio, } 0.97 ; 95 \% \text { CI, } 0.95 \text { to } 0.99 \text { ) } \\
\text { were lower in the future Joint } \\
\text { Commission-certified hospitals. }\end{array}$ & $\begin{array}{l}\text { Joint Commission Primary Stroke } \\
\text { Center-certified hospitals had } \\
\text { better outcomes than noncertified } \\
\text { hospitals even before the program } \\
\text { began. }\end{array}$ & 3 \\
\hline $\begin{array}{l}\text { Luder et al., } \\
2015\end{array}$ & 1 & $\begin{array}{l}\text { Community } \\
\text { pharmacy-based } \\
\text { transition of care } \\
\text { (TOC) program }\end{array}$ & $\begin{array}{l}\mathrm{CHF}, \mathrm{COPD} \text {, } \\
\text { and pneumonia }\end{array}$ & $\begin{array}{l}\text { Hospital } \\
\text { readmissions, } \\
\text { resolved } \\
\text { medication-related } \\
\text { problems, and } \\
\text { increased patient } \\
\text { satisfaction }\end{array}$ & $\begin{array}{l}20 \% \text { of patients in the usual care group } \\
\text { were admitted to the hospital within } 30 \\
\text { days compared to } 6.9 \% \text { of patients in the } \\
\text { intervention group }(p=.019)\end{array}$ & $\begin{array}{l}\text { Community pharmacies } \\
\text { successfully collaborated with } \\
\text { hospitals to develop a referral } \\
\text { process for TOC interventions. } \\
\text { Patients who received MTM } \\
\text { services from the pharmacist } \\
\text { experienced significantly fewer } \\
\text { readmissions than patients who } \\
\text { received usual care. }\end{array}$ & 2 \\
\hline $\begin{array}{l}\text { Maeng et al., } \\
2014\end{array}$ & 8 & Tele-monitoring & Heart failure & $\begin{array}{l}\text { Hospital } \\
\text { admission rates, } \\
\text { readmission rates, } \\
\text { and total cost of } \\
\text { care }\end{array}$ & $\begin{array}{l}\text { Significant reductions in probability of } \\
\text { all-cause admission (odds ratio [OR] } 0.77 \text {; } \\
p<.01 \text { ), 30-day and } 90 \text {-day readmission } \\
\text { (OR } 0.56,0.62 ; p<.05 \text { ), and cost of care } \\
(11.3 \% ; p<.05) \text {. }\end{array}$ & $\begin{array}{l}\text { These findings imply that } \\
\text { tele-monitoring can be an effective } \\
\text { add-on tool for managing elderly } \\
\text { patients with heart failure. }\end{array}$ & 3 \\
\hline Ota et al., 2013 & 3 & $\begin{array}{l}\text { Direct Telephonic } \\
\text { Communication in a } \\
\text { Heart Failure } \\
\text { Transitional Care } \\
\text { Program (HFTCP). }\end{array}$ & Heart failure & $\begin{array}{l}\text { 30-day } \\
\text { readmission }\end{array}$ & $\begin{array}{l}\text { Structured post-discharge follow up } \\
\text { protocols have been shown to help } \\
\text { prevent and reduce HF hospitalizations, } \\
\text { especially } 30 \text {-day readmissions. }\end{array}$ & $\begin{array}{l}\text { This program reduces unnecessary } \\
\text { hospitalizations and reduces costs } \\
\text { while giving patients the security } \\
\text { of having immediate access to } \\
\text { their provider. }\end{array}$ & 4 \\
\hline
\end{tabular}


Table 3 (continued.)

\begin{tabular}{|c|c|c|c|c|c|c|c|}
\hline Authors & Type & $\begin{array}{l}\text { Intervention } \\
\text { Description }\end{array}$ & Diagnosis & Outcome & Significance & Takeaway points & $\begin{array}{l}\text { Quality of } \\
\text { Evidence }\end{array}$ \\
\hline $\begin{array}{l}\text { Robbins et al., } \\
2014\end{array}$ & 11 & $\begin{array}{l}\text { Total Hip } \\
\text { Arthroplasty } \\
\text { (THA)-Accelerated } \\
\text { Rehabilitation (AR) } \\
\text { protocol }\end{array}$ & $\begin{array}{l}\text { Total hip } \\
\text { arthroplasty }\end{array}$ & $\begin{array}{l}\text { Early } \\
\text { mobilization, } \\
\text { length of stay, } \\
\text { complication, and } \\
\text { hospital } \\
\text { readmissions }\end{array}$ & $\begin{array}{l}\text { Accrelerated rehabilitation (AR) protocol } \\
\text { patients were more likely to be discharged home } \\
\text { and to have fewer postoperative complications } \\
\text { and rehospitalizations. Among } 16 \text { reported } \\
\text { readmission for the } 590 \text { patients included in this } \\
\text { retrospective review, only one was an AR } \\
\text { patient. }\end{array}$ & $\begin{array}{l}\text { A THA-AR protocol can decrease } \\
\text { LOS, influence discharge } \\
\text { disposition, and decrease the } \\
\text { likelihood of postoperative } \\
\text { complications and } \\
\text { rehospitalizations. }\end{array}$ & 4 \\
\hline $\begin{array}{l}\text { Roglieri et al., } \\
1997\end{array}$ & $\begin{array}{l}8,3 \\
4\end{array}$ & $\begin{array}{l}\text { A comprehensive } \\
\text { congestive heart } \\
\text { failure }(\mathrm{CHF}) \\
\text { disease } \\
\text { management } \\
\text { program }\end{array}$ & CHF & $\begin{array}{l}\text { Hospital } \\
\text { admission and } \\
\text { readmission rates, } \\
\text { length of stay, } \\
\text { total hospital } \\
\text { days, and } \\
\text { emergency room } \\
\text { utilization }\end{array}$ & $\begin{array}{l}\text { Overall, there were significantly reduced } \\
\text { admission and readmission rates for patients } \\
\text { with the pure CHF diagnosis; } 30 \text {-day and } \\
90 \text {-day readmission rates declined } 75 \% \text { ( } p= \\
.02 \text { ) and } 74 \%(p=.004) \text {, respectively. } \\
\text { Reductions were also seen in total hospital days } \\
\text { and emergency room utilization. }\end{array}$ & $\begin{array}{l}\text { A comprehensive disease } \\
\text { management program can reduce } \\
\text { healthcare utilization not only } \\
\text { among CHF patients in the program } \\
\text { but also among the entire managed } \\
\text { care plan population. }\end{array}$ & 3 \\
\hline $\begin{array}{l}\text { Rothberg et al., } \\
2010\end{array}$ & 5 & Antibiotic therapy & COPD & $\begin{array}{l}\text { Treatment failure, } \\
\text { inpatient } \\
\text { mortality, or } \\
\text { readmission for } \\
\text { acute } \\
\text { exacerbations of } \\
\text { COPD within } 30 \\
\text { days of discharge }\end{array}$ & $\begin{array}{l}\text { Of } 84,621 \text { patients, } 79 \% \text { received at least } 2 \\
\text { consecutive days of antibiotic treatment. } \\
\text { Treated patients were less likely than } \\
\text { non-treated patients to receive mechanical } \\
\text { ventilation after the second hospital day, and } \\
\text { had lower rates of readmission for acute } \\
\text { exacerbations of COPD }(7.91 \% ; 95 \% \text { CI, } \\
7.89 \%-7.94 \% \text { vs. } 8.79 \% ; 95 \% \text { CI, } \\
8.74 \%-8.83 \%)\end{array}$ & $\begin{array}{l}\text { Early antibiotic administration was } \\
\text { associated with improved outcomes } \\
\text { among patients hospitalized for } \\
\text { acute exacerbations of COPD } \\
\text { regardless of the risk of treatment } \\
\text { failure. }\end{array}$ & 3 \\
\hline $\begin{array}{l}\text { Ryan et al., } \\
2014\end{array}$ & 9 & $\begin{array}{l}\text { Quality } \\
\text { improvement (QI) } \\
\text { program }\end{array}$ & Heart failure & $\begin{array}{l}\text { The percentage of } \\
\text { patients who had } \\
\text { preventable } \\
\text { readmissions in } \\
\text { each year }\end{array}$ & $\begin{array}{l}\text { The } 30 \text {-day readmission rate decreased } 28 \%(p \\
=.04 \text { ) following the initiative of Quality } \\
\text { Improvement program }\end{array}$ & $\begin{array}{l}\text { This study identified } 20 \% \text { to } 30 \% \text { of } \\
2008 \text { readmissions preventable } \\
\text { based on chart review by multiple } \\
\text { providers having different clinical } \\
\text { backgrounds. This range contains } \\
\text { the actual } 28 \% \text { readmission } \\
\text { reduction rate that occurred } \\
\text { following the UCONN HF QI } \\
\text { program (intervention). Preventable } \\
\text { readmissions were } \\
\text { less common after the QI program } \\
\text { was in place. }\end{array}$ & 3 \\
\hline $\begin{array}{l}\text { Sales et al., } \\
2014\end{array}$ & $\begin{array}{l}3,6 \\
4\end{array}$ & $\begin{array}{l}\text { Pharmacologic } \\
\text { education by a } \\
\text { trained volunteer; } \\
\text { receiving dietary, } \\
\text { follow-up calls }\end{array}$ & $\mathrm{CHF}$ & $\begin{array}{l}\text { 30-day } \\
\text { readmission }\end{array}$ & $\begin{array}{l}\text { The intervention arm (Arm A) patients, who } \\
\text { received dietary and pharmacologic education } \\
\text { by a trained volunteer, had decreased 30-day } \\
\text { readmissions ( } 7 \% \text { vs. 19\%; } p<.05 \text { ) with a } \\
\text { relative risk reduction (RRR) of } 63 \% \text { and an } \\
\text { absolute risk reduction (ARR) of } 12 \% \text {. }\end{array}$ & $\begin{array}{l}\text { Utilizing trained volunteer staff to } \\
\text { improve patient education and } \\
\text { engagement might be an efficient } \\
\text { and low-cost intervention to reduce } \\
\text { CHF readmissions. }\end{array}$ & 1 \\
\hline $\begin{array}{l}\text { Scotti et al., } \\
2015\end{array}$ & 11 & $\begin{array}{l}\text { Percutaneous } \\
\text { ventricular assist } \\
\text { devices (pVADs) } \\
\text { and extracorporeal } \\
\text { membrane } \\
\text { oxygenation } \\
\text { (ECMO) }\end{array}$ & $\begin{array}{l}\text { Cardiogenic } \\
\text { Shock (CS) }\end{array}$ & $\begin{array}{l}\text { 30- and 90-day } \\
\text { readmissions, } \\
\text { associated length } \\
\text { of stay (LOS), and } \\
\text { costs }\end{array}$ & $\begin{array}{l}\text { At } 90 \text { days after initial hospitalization, the } \\
\text { readmission rates in the pVAD and ECMO } \\
\text { cohorts were } 38.7 \%(200 / 517) \text { and } 53.0 \% \\
\text { (70/132), respectively. Overall, pVAD was } \\
\text { associated with a } 27.1 \% \text { reduction in } \\
\text { readmission }(p=.004) \text {. }\end{array}$ & $\begin{array}{l}\text { Increased adoption of pVAD, as a } \\
\text { technology to support patients in } \\
\text { cardiogenic shock, may help } \\
\text { hospitals deliver greater value to } \\
\text { both government and commercial } \\
\text { payers. }\end{array}$ & 3 \\
\hline $\begin{array}{l}\text { Snider et al., } \\
2015\end{array}$ & 11 & $\begin{array}{l}\text { Oral nutritional } \\
\text { supplementation } \\
\text { (ONS) }\end{array}$ & COPD & $\begin{array}{l}\text { Readmission, } \\
\text { length of stay } \\
\text { (LOS), and cost }\end{array}$ & $\begin{array}{l}\text { ONS use was associated with longer LOS ( } 8.7 \\
\text { days vs. } 6.9 \text { days, } p<.0001) \text {, higher } \\
\text { hospitalization cost }(\$ 14,223 \text { vs. } \$ 9,340, p< \\
.0001) \text {, and lower readmission rates }(24.8 \% \text { vs. } \\
26.6 \%, p=.0116) \text {. }\end{array}$ & $\begin{array}{l}\text { ONS may be associated with } \\
\text { reduced LOS, hospitalization cost, } \\
\text { and readmission risk in hospitalized } \\
\text { Medicare patients with COPD. }\end{array}$ & 4 \\
\hline $\begin{array}{l}\text { Stauffer et al., } \\
2011\end{array}$ & 2,4 & $\begin{array}{l}\text { Nurse-led } \\
\text { transitional care } \\
\text { programs }\end{array}$ & Heart failure & $\begin{array}{l}\text { 30-day all-cause } \\
\text { readmission rate, } \\
\text { length of stay, and } \\
60 \text {-day (from } \\
\text { admission) direct } \\
\text { cost }\end{array}$ & $\begin{array}{l}\text { The intervention significantly reduced adjusted } \\
\text { 30-day readmission rates by } 48 \% \text { during the } \\
\text { post-intervention period. The intervention, } \\
\text { however, had little effect on length of stay or } \\
\text { total } 60 \text {-day direct costs. }\end{array}$ & $\begin{array}{l}\text { Preliminary results suggest that } \\
\text { transitional care programs reduce } \\
\text { 30-day readmission rates for } \\
\text { patients with heart failure. This } \\
\text { underscores the potential of the } \\
\text { intervention to be effective in a } \\
\text { real-world setting, but payment } \\
\text { reform may be required for the } \\
\text { intervention to be financially } \\
\text { sustainable by hospitals. }\end{array}$ & 2 \\
\hline
\end{tabular}

Note. The type of interventions are defined in Table 2

Given the heterogeneous ways in which interventions were implemented among the studies, we determined whether interventions were implemented as a single construct or op- erationalized in a bundled fashion. We found that 16 of the 45 studies examined bundled interventions (i.e. interventions that incorporated more than one strategy in tandem), while 
29 studied the implementation of a single intervention. Educational strategies were more frequently bundled with telephone follow-up, medication reconciliation, and discharge planning, while collaboration was more frequently bundled with in-hospital management and guideline implementation, indicating more of a focus on internal management. Generally, a higher proportion of the singularly-implemented interventions found beneficial associations on readmission (16 of 29 studies [55.2\%]) than the bundled interventions (6 of 16 studies [37.5\%]).

When looking at intervention strategies and effectiveness by diagnosis type, we found that $50.7 \%$ of included studies examined readmission among heart failure patients (see Table
4). Of the 40 examined interventions among heart failure patients, $21(52.5 \%)$ of them found that interventions were beneficial in reducing readmission. We note that tele-monitoring was the most consistent intervention with beneficial results in readmission reductions among heart failure patients. Despite the common and pervasive occurrence of sepsis among hospitalized individuals, we found that no studies had examined interventions and short-term readmission among sepsis patients. The evidence-base for intervention efficacy among THA/TKA $(n=2)$, and stroke patients $(n=2)$ is also quite limited, suggesting that future research should seek to fill these gaps in knowledge in order to improve outcomes for these patients.

Table 4. Number of studies by diagnoses and interventions

\begin{tabular}{|c|c|c|c|c|c|c|c|c|}
\hline & $\begin{array}{l}\text { Heart } \\
\text { Failure }\end{array}$ & AMI & COPD & $\begin{array}{l}\text { Bronchiti/ } \\
\text { Asthma }\end{array}$ & $\begin{array}{l}\text { Pneumonia and Other } \\
\text { Respiratory Tract } \\
\text { Infections }\end{array}$ & Sepsis & Stroke & $\begin{array}{l}\text { Hip or Knee Joint } \\
\text { Replacement }\end{array}$ \\
\hline Collaboration & $5(3)$ & 0 & $2(1)$ & $2(0)$ & $2(1)$ & 0 & 0 & 0 \\
\hline Home visits & $1(1)$ & 0 & 0 & 0 & 0 & 0 & 0 & 0 \\
\hline $\begin{array}{l}\text { Telephone } \\
\text { follow-up }\end{array}$ & $4(3)$ & 0 & $1(0)$ & 0 & 0 & 0 & 0 & 0 \\
\hline Education & $8(3)$ & 0 & $3(1)$ & 0 & 0 & 0 & 0 & 0 \\
\hline $\begin{array}{l}\text { Medication } \\
\text { reconciliation }\end{array}$ & $2(1)$ & $1(1)$ & $3(3)$ & 0 & $2(1)$ & 0 & 0 & 0 \\
\hline $\begin{array}{l}\text { Discharge } \\
\text { planning }\end{array}$ & $5(2)$ & $1(1)$ & $1(1)$ & 0 & $1(1)$ & 0 & 0 & 0 \\
\hline $\begin{array}{l}\text { Follow-up } \\
\text { appointment }\end{array}$ & $1(1)$ & 0 & 0 & 0 & 0 & 0 & 0 & 0 \\
\hline Tele-monitoring & $5(3)$ & $1(0)$ & 0 & 0 & $1(0)$ & 0 & 0 & 0 \\
\hline $\begin{array}{l}\text { Guideline } \\
\text { implementation }\end{array}$ & $3(1)$ & 0 & $1(0)$ & $1(0)$ & $1(1)$ & 0 & $1(1)$ & $1(1)$ \\
\hline Rehabilitation & $1(0)$ & 0 & 0 & 0 & 0 & 0 & $1(1)$ & 0 \\
\hline $\begin{array}{l}\text { Clinical, medical } \\
\text { device }\end{array}$ & $2(1)$ & 0 & $3(1)$ & $1(0)$ & 0 & 0 & 0 & $1(1)$ \\
\hline $\begin{array}{l}\text { In-hospital } \\
\text { management unit }\end{array}$ & $3(2)$ & $2(1)$ & $1(1)$ & $2(0)$ & $2(1)$ & 0 & 0 & 0 \\
\hline $\begin{array}{l}\text { Total number of } \\
\text { interventions by } \\
\text { diagnosis }\end{array}$ & $40(21)$ & $5(3)$ & $15(8)$ & $6(0)$ & $9(5)$ & 0 & $2(2)$ & $2(2)$ \\
\hline
\end{tabular}

Note. The numbers in parenthesis indicate the number of interventions with positive impacts on 30-day readmissions. The total number of interventions by diagnosis (79) is greater than the number of interventions (66) in Table 1 because some articles include analyses of more than one diagnosis

\section{Discussion}

In this systematic review paper we first report that the number of studies focused in readmission reduction interventions has increased dramatically since 2000 , adding to our knowledge of effective interventions and our ability to follow more evidence-based strategies to reduce hospital readmissions. Successful interventions targeted towards reducing readmission rates are presented and discussed next. One important observation is that from an organizational point of view, it is difficult to isolate the effect of single interventions as these interventions are often executed in bundles. For example, we found that educational strategies were more frequently bundled with telephone follow-up, medication reconciliation, and discharge planning. Due to the bundled nature of interventions, it is very difficult to design a study to evaluate the effect of one single intervention in a health care setting today.

Another key consideration in designing interventions to reduce readmission rates is disease category and patient population segments. Both patient education and collaboration 
type interventions show mixed results and seem to be highly dependent on disease and setting. For example, collaboration interventions demonstrate significant positive results for patients with heart disease, which is also the most studied disease type. These mixed results lead us to conclude that for readmission reduction interventions to be successful, they need to be targeted towards specific patient segments and disease categories. Furthermore, the success of each of these initiatives may be primarily patient-driven. Shared accountability for clinical outcomes between patients and healthcare teams represents an evolving and increasingly important concept in healthcare practice today. This paradigm is commonly described in the context of values-based medicine, a framework for clinical practice, which requires true partnerships between patients, providers and health systems to optimize healthcare outcomes.

Finally, future research on readmission reduction interventions should consider including additional context variables, such as socioeconomic factors, in readmission reduction studies and prediction models. We found that most studies did not consider income, insurance status, or provider characteristics in their studies. Overall, our findings indicate a major opportunity for researchers to pursue studies that employ tools that provide additional context and specificity so as to better inform the execution of interventional strategies in healthcare organizations.

Our study has noteworthy limitations. First, it is possible that our search protocol neglected to include some studies that may have been worthy of inclusion. To minimize this possibility, we consulted with a systematic review librarian, experimented with various search terms, and attuned our approach to err on the side of inclusion during the first phase of our search. Second, our sample size of 45 articles limited our ability to conduct complex analyses. This is especially true given that so many interventions are understudied as they relate to specific diagnoses. As health care delivery organizations and professionals continue to seek ways in which to improve quality of care, and by extension, population health, future work should further seek to identify the significant predictors of successful interventions as they relate to stemming unplanned hospitalizations.

\section{Conclusions}

This study reveals that readmission reduction interventions are executed in bundles and have mixed results based on patient population and diagnosis. Future research should consider additional context and specificity so as to better inform the successful readmission reduction strategies in healthcare organizations.

\section{ACKNOWLEDGEMENTS}

The preparation of this article is based on work supported by the National Science Foundation (NSF) under grant no. IIP-0832439. Findings and conclusions in this material are those of the authors and do not necessarily reflect the views of the NSF.

\section{CONFLICTS OF INTEREST Disclosure}

The authors declare they have no conflicts of interest.

\section{REFERENCES}

[1] Lichtman JH, Leifheit-Limson EC, Jones SB, et al. Predictors of hospital readmission after stroke: a systematic review. Stroke. 2010; 41(11): 2525-2533. PMid: 20930150. https ://doi.org/10.116 1/STROKEAHA. 110.599159

[2] McIlvennan CK, Eapen ZJ, Allen LA. Hospital readmissions reduction program. Circulation. 2015; 131(20): 1796-1803. PMid: 25986448. https://doi.org/10.1161/CIRCULATIONAHA .114 .010270

[3] Fingar K, Washington R. Trends in Hospital Readmissions for Four High-Volume Conditions, 2009-2013. 2015. Available from: https://www.hcup-us.ahrq.gov/reports/statbriefs/sb 196-Readmissions-Trends-High-Volume-Conditions.jsp

[4] Centers for Medicare \& Medicaid Services. Hospital Compare. 2016. Available from: https://www.cms.gov/medicare/quality-i nitiatives-patient-assessment-instruments/hospita lqualityinits/hospitalcompare.html

[5] Boccuti C, Casillas G. Aiming for fewer hospital u-turns: The Medicare Hospital Readmission Reduction Program. 2017. Available from: http://kff.org/medicare/issue-brief/aiming-for -fewer-hospital-u-turns-the-medicare-hospital-rea dmission-reduction-program/view/footnotes/\#footnot e-211172-3

[6] Desai NR, Ross JS, Kwon JY, et al. Association Between Hospital Penalty Status Under the Hospital Readmission Reduction Program and Readmission Rates for Target and Nontarget Conditions. JAMA. 2016; 316(24): 2647-2656. PMid: 28027367. https : //doi.org/10.1001/jama.2016.18533

[7] Hospital Readmission Reduction Program, Patient Protection and Affordable Care Act, §3025. 2010.

[8] Centers for Medicare \& Medicaid Services. Readmissions Reduction Program (HRRP). 2016. Available from: https://www.cms.gov/ medicare/medicare-fee-for-service-payment/acutein patientpps/readmissions-reduction-program.html

[9] Adamina M, Kehlet H, Tomlinson GA, et al. Enhanced recovery pathways optimize health outcomes and resource utilization: a metaanalysis of randomized controlled trials in colorectal surgery. Surgery. 2011; 149(6): 830-840. PMid: 21236454. https://doi.org/10 $.1016 /$ j.surg. 2010.11 .003

[10] Chambers MC, El-Othmani MM, Anoushiravani AA, et al. Reducing 30-day Readmission After Joint Replacement. Orthop Clin North 
Am. 2016; 47(4): 673-680. PMid: 27637653. https://doi.org/ $10.1016 / \mathrm{j} . \mathrm{ocl} .2016 .05 .014$

[11] Del Sindaco D, Pulignano G, Minardi G, et al. Two-year outcome of a prospective, controlled study of a disease management programme for elderly patients with heart failure. Journal of Cardiovascular Medicine (Hagerstown, Md). 2007; 8(5): 324-329. PMid: 17443097. https://doi.org/10.2459/JCM.0b013e32801164cb

[12] Benbassat J, Taragin MI. The effect of clinical interventions on hospital readmissions: a meta-review of published meta-analyses. Israel Journal of Health Policy Research. 2013; 2(1): 1. PMid: 23343012. https://doi .org/10.1186/2045-4015-2-1

[13] Slyer JT, Concert CM, Eusebio AM, et al. A systematic review of the effectiveness of nurse coordinated transitioning of care on readmission rates for patients with heart failure. JBI Library of Systematic Reviews. 2011; 9(15): 464-490. https ://doi .org/10.11124/0 1938924-201109150-00001

[14] Chiu WK, Newcomer R. A systematic review of nurse-assisted case management to improve hospital discharge transition outcomes for the elderly. Prof Case Manag. 2007; 12(6): 330-336; quiz 337338. PMid: 18030153. https://doi.org/10.1097/01. PCAMA . 0 $000300406.15572 . \mathrm{e} 2$

[15] Rochester-Eyeguokan CD, Pincus KJ, Patel RS, et al. The Current Landscape of Transitions of Care Practice Models: A Scoping Review. Pharmacotherapy. 2016; 36(1): 117-133. PMid: 26799353. https://doi.org/10.1002/phar.1685

[16] Caplan GA, Sulaiman NS, Mangin DA, et al. A meta-analysis of "hospital in the home". The Medical Journal of Australia. 2012; 197(9): 512-519. PMid: 23121588. https://doi.org/10.5694/mja12. 10480

[17] Feltner C, Jones CD, Cene CW, et al. Transitional Care Interventions To Prevent Readmissions for People With Heart Failure. In: AHRQ Comparative Effectiveness Reviews, ed. Rockville (MD): Agency for Healthcare Research and Quality (US); 2014.

[18] Christensen M, Lundh A. Medication review in hospitalised patients to reduce morbidity and mortality. Cochrane Database Systematic Review. 2013(2).

[19] Cockayne S, Pattenden J, Worthy G, et al. Nurse facilitated Selfmanagement support for people with heart failure and their family carers (SEMAPHFOR): a randomised controlled trial. International Journal of Nursing Studies. 2014; 51(9): 1207-1213. PMid: 24508285. https://doi.org/10.1016/j.ijnurstu.2014.01.010

[20] Fredericks S, Yau T. Clinical effectiveness of individual patient education in heart surgery patients: A systematic review and metaanalysis. International Journal of Nursing Studies. 2017; 65: 4453. PMid: 27842225. https://doi.org/10.1016/j.ijnurstu .2016 .11 .001

[21] Pandor A, Thokala P, Gomersall T, et al. Home telemonitoring or structured telephone support programmes after recent discharge in patients with heart failure: systematic review and economic evaluation. Health Technology Assessment (Winchester, England). 2013; 17(32): 1-207, v-vi. https ://doi .org/10.3310/hta17320

[22] McMartin K. Discharge planning in chronic conditions: an evidencebased analysis. Ontario Health Technology Assessment Series. 2013; 13(4): $1-72$

[23] Fox MT, Persaud M, Maimets I, et al. Effectiveness of early discharge planning in acutely ill or injured hospitalized older adults: a systematic review and meta-analysis. BMC Geriatr. 2013; 13: 70. PMid: 23829698. https://doi.org/10.1186/1471-2318-13-70

[24] Braet A, Weltens C, Sermeus W. Effectiveness of discharge interventions from hospital to home on hospital readmissions: a systematic review. JBI Database of Systematic Reviews and Im- plementation Reports. 2016; 14(2): 106-173. PMid: 27536797. https://doi.org/10.11124/jbisrir-2016-2381

[25] Puhr MI, Thompson HJ. The Use of Transitional Care Models in Patients With Stroke. The Journal of Neuroscience Nursing: Journal of the American Association of Neuroscience Nurses. 2015; 47(4): 223-234. PMid: 25906245 . https://doi.org/10.1097/JNN.00 00000000000143

[26] Lehnbom EC, Stewart MJ, Manias E, et al. Impact of medication reconciliation and review on clinical outcomes. Ann Pharmacother 2014; 48(10): 1298-1312. PMid: 25048794. https ://doi .org/10 $.1177 / 1060028014543485$

[27] Christensen M, Lundh A. Medication review in hospitalised patients to reduce morbidity and mortality. Cochrane Database Syst Rev. 2016(2).

[28] Kane RL, Homyak P, Bershadsky B, et al. The effects of a variant of the program for all-inclusive care of the elderly on hospital utilization and outcomes. Journal of the American Geriatrics Society. 2006; 54(2): 276-283. PMid: 16460379. https ://doi .org/10.1111/j. 1532-5415.2005.00581. $\mathrm{x}$

[29] Naylor MD, Brooten D, Campbell R, et al. Comprehensive discharge planning and home follow-up of hospitalized elders: a randomized clinical trial. JAMA : the Journal of the American Medical Association. 1999; 281(7): 613-620. PMid: 10029122. https://doi.org/10.1001/jama.281.7.613

[30] Crotty M, Rowett D, Spurling L, et al. Does the addition of a pharmacist transition coordinator improve evidence-based medication management and health outcomes in older adults moving from the hospital to a long-term care facility? Results of a randomized, controlled trial. Am J Geriatr Pharmacother. 2004; 2(4): $257-$ 264. PMid: 15903284. https ://doi.org/10.1016/j.amjophar m.2005.01.001

[31] Imazio M, Cotroneo A, Gaschino G, et al. Management of heart failure in elderly people. International Journal of Clinical Practice. 2008; 62(2): 270-280. PMid: 18070044. https ://doi .org/10.1 $111 / j .1742-1241.2007 .01583 . x$

[32] Jovicic A, Holroyd-Leduc JM, Straus SE. Effects of self-management intervention on health outcomes of patients with heart failure: a systematic review of randomized controlled trials. BMC Cardiovasc Disord. 2006; 6: 43. PMid: 17081306. https://doi.org/10.118 6/1471-2261-6-43

[33] Schoenfeld AJ, Zhang X, Grabowski DC, et al. Hospital-skilled nursing facility referral linkage reduces readmission rates among Medicare patients receiving major surgery. Surgery. 2016; 159(5): 1461-1468. PMid: 26830069. https://doi.org/10.1016/j.su rg. 2015.12.012

[34] Renaudin P, Boyer L, Esteve MA, et al. Do pharmacist-led medication reviews in hospitals help reduce hospital readmissions? A systematic review and meta-analysis. British Journal of Clinical Pharmacology. 2016; 82(6): 1660-1673. PMid: 27511835. https://doi.org/10.1111/bcp.13085

[35] Kansagara D, Chiovaro JC, Kagen D, et al. So many options, where do we start? An overview of the care transitions literature. J Hosp Med. 2016; 11(3): 221-230. PMid: 26551918. https : //doi.org/10.1002/jhm. 2502

[36] Moher D, Shamseer L, Clarke M, et al. Preferred reporting items for systematic review and meta-analysis protocols (PRISMA-P) 2015 statement. Systematic Reviews. 2015; 4(1): 1. PMid: 25554246. https://doi.org/10.1186/2046-4053-4-1

[37] Galinanes EL, Dombroviskiy VY, Hupp CS, et al. Evaluation of readmission rates for carotid endarterectomy versus carotid artery stenting in the US Medicare population. Vasc Endovascular Surg. 
2014; 48(3): 217-223. PMid: 24407509. https ://doi.org/10.1 $177 / 1538574413518120$

[38] Sueta CA, Schenck A, Chowdhury M, et al. Effect of angiotensinconverting enzyme inhibitor therapy on 30-day outcome in patient $>$ or $=65$ years of age with chronic congestive heart failure. Am J Cardiol. 2000; 86(10): 1151-1153, A1159.

[39] Dragu R, Behar S, Sandach A, et al. Should primary percutaneous coronary intervention be the preferred method of reperfusion therapy for patients with renal failure and ST-elevation acute myocardial infarction? Am J Cardiol. 2006; 97(8): 1142-1145. PMid: 16616015. https://doi.org/10.1016/j.amjcard.2005.11.028

[40] Lindenauer PK, Normand SL, Drye EE, et al. Development, validation, and results of a measure of 30-day readmission following hospitalization for pneumonia. J Hosp Med. 2011; 6(3): 142-150. PMid: 21387551. https://doi.org/10.1002/jhm. 890

[41] Davies S, Saynina O, Schultz E, et al. Implications of metric choice for common applications of readmission metrics. Health Ser- vices Research. 2013; $48(6$ Pt 1): 1978-1995. PMid: 23742056. https://doi.org/10.1111/1475-6773.12075

[42] Mull HJ, Chen Q, O'Brien WJ, et al. Comparing 2 methods of assessing 30-day readmissions: what is the impact on hospital profiling in the veterans health administration? Medical Care. 2013; 51(7): 589-596. PMid: 23604016. https ://doi.org/10.1097/MLR. ob 013e31829019a4

[43] Kash BA, Baek J, Davis E, et al. Review of successful hospital readmission reduction strategies and the role of health information exchange. International Journal of Medical Informatics. 2017; 104: 97-104. PMid: 28599821. https://doi.org/10.1016/j.ijme dinf. 2017.05.012

[44] Kash BA, Baek J, Cheon O, et al. Successful hospital readmission reduction initiatives: Top five strategies to consider implementing today. Journal of Hospital Administration. 2018; 7(6): 16-23. https://doi.org/10.5430/jha.v7n6p16

[45] OCEBM Levels of Evidence Working Group. The Oxford Levels of Evidence 2. Oxford Centre for Evidence-Based Medicine. 